\title{
Instrumental performance and time between reinforcements: Intimate relation to learning or memory retrieval?
}

\author{
E. J. CAPALDI, SUZAN ALPTEKIN, and KIMBERLY M. BIRMINGHAM \\ Purdue University, West Lafayette, Indiana
}

\begin{abstract}
According to scalar expectancy theory (SET), instrumental performance is determined by the ratio of the time between reinforcements in the trial $\left(T_{\mathrm{T}}\right)$ to the overall time between reinforcements $\left(T_{\mathrm{O}}\right)$. Groups for which the $T_{\mathrm{O}} \mid T_{\mathrm{T}}$ ratio is the same should perform similarly. According to the sequentialmemory view, the memory of nonreward becomes a signal for reward, and thereby promotes strong responding, when that memory is retrieved on a reward trial. In each of three runway investigations employing rats in a runway, two groups were compared that had the same $T_{\mathrm{O}} \mid T_{\mathrm{T}}$ ratio but that differed in the tendency to retrieve the memory of nonreward on a rewarded trial. In each investigation faster running on critical nonrewarded trials was associated with the group having the stronger tendency to retrieve the memory of nonreward on a rewarded trial. These findings are consistent with the predictions of the sequential-memory view, as well as with certain earlier findings, but are inconsistent with SET. It was indicated that the groups compared here were matched along a considerable number of dimensions-an unprecedented number for a varied reward investigation.
\end{abstract}

The three experiments reported here were explicitly designed as a test of two views of how time between reinforcements affects instrumental learning and performance, views that are quite dissimilar. The two views are scalar expectancy theory, or SET (see, e.g., Gibbon, Farrell, Locurto, Duncan, \& Terrace, 1980) and the sequentialmemory view (e.g., Capaldi, 1994). Available reward schedule experiments that support one of these views do not necessarily disconfirm the other. A critical test of the two views was attempted here.

Two SET propositions are that timing and learning are intimately related and that goal-produced memories do not regulate instrumental responding (see, e.g., Gibbon \& Balsam, 1981; Gibbon et al., 1980). Contrary to SET, the sequential view suggests that time-related cues, although possibly quite salient, are not particularly special or unique and that instrumental behavior is strongly controlled by goal-produced memories (see, e.g., Capaldi, 1994). The three experiments reported here are relevant to both these theories.

Gibbon et al. suggested that SET, which has been applied to Pavlovian phenomena, can also be applied to instrumental partial reward runway data. More recently, SET has been applied to various other instrumental learning phenomena (see, e.g., Gallistel, 1994). According to SET, performance in instrumental tasks, including runway tasks, is determined by the ratio of time between reinforcements in the trial $\left(T_{\mathrm{T}}\right)$ to the total or overall time between reinforcements $\left(T_{\mathrm{O}}\right)$. Groups for which this $T_{\mathrm{O}} \mid T_{\mathrm{T}}$

Correspondence should be addressed to: E. J. Capaldi, Department of Psychology, Purdue University, 1364 Psychology Building, West Lafayette, IN 47907-1364 (e-mail: julie@psych.purdue.edu). ratio is the same, however arrived at, should perform similarly both in acquisition and in extinction. Gibbon et al. asserted that this prediction, which was confirmed in an autoshaping situation varying percentage of reward and intertrial interval, also applied to instrumental runway tasks and that it was incompatible with the memorysequential view, or more generally with views suggesting that internal, goal-related stimuli regulate instrumental responding.

According to the sequential view, if a memory of a particular reward event - say, the memory of nonreward - is retrieved on a rewarded trial, it will acquire the capacity to promote vigorous responding. With this proposition in mind, we will briefly outline the strategy employed here to test SET and the memory sequential view. In each of three investigations, two groups were compared that at the outset of timing had identical $T_{\mathrm{O}} \mid T_{\mathrm{T}}$ ratios. However, in one group, the memory of nonreward was made to be a better predictor of reward than in the other group. Thus the sequential-memory view predicts, contrary to SET, that the two groups should differ. The group for which the memory of nonreward is the better predictor of reward should be the faster on certain nonrewarded trials.

The memory of nonreward should be better retrieved on a rewarded trial, and thus become a better predictor of reward, to the extent that the cues that accompany the nonrewarded storage trial also occur on the subsequent rewarded retrieval trial. In Experiments 1 and 2 we employed intertrial interval (ITI) as the critical retrieval cue, with runway brightness serving that function in Experiment 3. In Experiments 1 and 2, the ITI preceding the nonrewarded storage trial was either the same or different from the ITI preceding the rewarded retrieval trial: In Experiment 3 , runway brightness was the same or different 
in connection with the two sorts of trials. Retrieval of the memory of nonreward on the rewarded trial should be better when similar rather than different stimuli accompany the two sorts of trials.

Consistent with the sequential view, it has been reported in a number of experiments that performance following nonrewarded trials is poorer in groups for which the critical storage and retrieval ITIs are different rather than the same (e.g., Capaldi, Nawrocki, Miller, \& Verry, 1986). These findings, however, are not necessarily incompatible with SET: In all but one of those investigations the groups compared had different $T_{\mathrm{O}} \mid T_{\mathrm{T}}$ ratios. Where the $T_{\mathrm{O}} \mid T_{\mathrm{T}}$ ratio was the same (Capaldi et al., 1986, Experiment 5), one of the groups did and the other did not receive a changed ITI within a day's trials. The changed ITI was sometimes shorter and sometimes longer than the other ITIs of the day.

The failure of the above-cited investigations to provide a critical test of SET and memory-retrieval views is due, we suggest, to the fact that each group in each investigation was trained under a single reward schedule (i.e., a schedule of partial reward). Under the single reward schedule procedure, varying the storage and retrieval ITIs almost inevitably introduces differences in the $T_{\mathrm{O}} \mid T_{\mathrm{T}}$ ratio. It is possible to avoid that problem, and to provide a critical test of SET and memory-retrieval views, by training groups, as in the present investigations, under two different reward schedules, a partial reward schedule and a consistent reward schedule. Employing the two-schedule procedure made it possible here to keep the $T_{\mathrm{O}} \mid T_{\mathrm{T}}$ ratio the same for all groups while affecting the retrieval of critical memories as follows. First, each group received a number of trials for which the storage and retrieval ITIs were the same. However, each group also received storage and retrieval trials that differed either in ITI or in brightness. The different storage and retrieval trials occurred in connection with the partial reward schedule in one group and in connection with the consistent reward schedule in the other group. As will be shown in detail later, SET predicts that the two groups in each experiment should not differ, consistent with the autoshaping findings reported by Gibbon et al. (1980). The memory view predicts that relatively slow running will develop on Trials 2 and 3 of the partial reward schedule when the different storage and retrieval trials occur in that schedule rather than in the consistent reward schedule.

In Experiment 1, the critical retrieval ITI was longer than the storage ITI. In Experiment 2, the opposite was the case. In Experiment 3, manipulation of the critical storage and retrieval trials occurred in connection with runway brightness rather than ITI. According to the sequential view, brightness cues should affect performance, as do time-related cues.

\section{EXPERIMENT 1}

Each of the two groups employed in Experiment 1 received each day a four-trial schedule of partial reward and a four-trial schedule of consistent reward so that the $T_{\mathrm{O}} \mid T_{\mathrm{T}}$ ratio was the same at the beginning of training. In each group the storage ITI (that preceding Trial 3) and the retrieval ITI (that preceding Trial 4) were the same in one of the schedules and different in the other schedule. The storage and retrieval ITIs differed in the partial schedule in one group (Group LP) and in the consistent schedule in the other group (Group LC). It was in the partial schedule that the memory of nonreward stored on Trial 3 could become a signal for reward on Trial 4 . This means that the memory of nonreward was more poorly retrieved on Trial 4 in Group LP (different storage and retrieval ITIs) than in Group LC (same storage and retrieval ITIs). Thus the sequential-memory view predicts that Group LP will run more slowly than Group LC on certain of the nonrewarded trials. The partial reward schedule always consisted of three nonrewarded trials followed by a rewarded trial. The consistent reward schedule consisted of four rewarded trials. On half the days the partial schedule occurred first and on half the days, second. The interschedule interval (ISI) was about $18 \mathrm{~min}$. All ITIs of both schedules were about $20 \mathrm{sec}$ except one, that elapsing between Trials 3 and 4 of one of the schedules. That ITI, which was about $5 \mathrm{~min}$, occurred in connection with the partial schedule in Group LP and the consistent schedule in Group LC. Thus, the critical storage and retrieval ITIs for the memory of nonreward differed in Group LP but were the same in Group LC. On each day of training following the initial rewarded trial of the day, each group received five 20sec ITIs, one 5-min ITI, and one 18-min ISI. Since the two groups were equated for ITI and ISI, it follows that if early in training the two groups run equally rapidly in the trial, they would have the same $T_{\mathrm{O}} \mid T_{\mathrm{T}}$ ratio.

As indicated, according to the sequential view, retrieval on Trial 4 of the memory stored on Trial 3 should be poor in that schedule in which the storage and retrieval ITIs differed. This would be the partial reward schedule in Group LP and the consistent reward schedule in Group LC.

\section{Method}

Subjects. The subjects were 8 experimentally naive male albino rats purchased from the Holtzman Co., Madison, WI. They were 90 days old on arrival at the laboratory.

Apparatus. The apparatus was a straight gray runway, $136.3 \mathrm{~cm}$ long, $9.1 \mathrm{~cm}$ wide, and enclosed by $9.2-\mathrm{cm}$ sides; it was covered by a wire-mesh top on a hinged frame. The startbox and goalbox were $23.2 \mathrm{~cm}$ and $30.1 \mathrm{~cm}$ long, respectively, and were closed off by metal guillotine doors. Raising the startbox door started a completely silent 0.01 -sec digital clock, which was stopped when a photobeam located $106.3 \mathrm{~cm}$ beyond the startbox door and $2.2 \mathrm{~cm}$ in front of the goal cup was broken by the rat. Food (45-mg Noyes pellets) could be placed in the goal cup, which measured $4.0 \mathrm{~cm}$ in diameter and $1.5 \mathrm{~cm}$ in depth, cut into a wood block $(4 \times 4.5 \times 9 \mathrm{~cm})$ located at the end of the runway. Baiting of an adjacent goalbox occurred on nonrewarded trials. Each experiment reported here was run by an undergraduate student under the supervision of a graduate student. The undergraduate had no idea of the purpose of the experiment, which was explained after the experiment was over.

Pretraining. On arrival at the laboratory, all rats were caged individually and were given ad-lib food and water for 17 days. They were then placed on deprivation, consisting of $18 \mathrm{~g}$ of Wayne Ro- 
dent Blox each day. On Days 1-7 of deprivation, each rat was handled for about $1 \mathrm{~min}$. On Day 8 each rat was fed eight $45-\mathrm{mg}$ Noyes pellets in the home cage. On Days 8-10, each rat was given 3 min of exploration in the alley with 8 pellets scattered throughout the alley. Also, at the 1.5-min mark of alley exploration each day, the guillotine doors were lowered and raised to acquaint the rat with this noise. After all rats had received alley exploration they were returned to their home cages and fed the daily ration (minus any food pellets eaten).

On Days 11-13, each rat received two trials that terminated in food reward $(R)$, consisting of eight $45-\mathrm{mg}$ Noyes pellets. All rats received the first $R$ trial before any rat received the second $R$ trial. Immediately after eating the pellets, the rat was removed from the goalbox.

Experimental training. Experimental training began on Day 14 and continued for 18 days. Trials terminated in either an eight-pellet reward $(R)$ or 15-sec nonrewarded confinement in any empty goalbox. Each animal received each day two schedules of four trials, a schedule of consistent reward, all $\mathrm{R}$ trials, and a schedule of partial reward. The partial reward schedule always consisted of three successive nonrewarded, or $\mathrm{N}$ trials, followed by an $\mathrm{R}$ trial. For all rats, all ITIs were approximately $20 \mathrm{sec}$ except one that was approximately 5 min. The long ITI always occurred between Trials 3 and 4 . In Group LP, the 5-min ITI occurred between Trials 3 and 4 of the partial schedule. In Group LC, the long ITI occurred between Trials 3 and 4 of the consistent schedule.

The order of presenting the partial schedule $(P)$ and the consistent schedule (C) over days was as follows: Day 1, C then P; Day 2, $P$ then C; Day 3, P then C; and Day 4, C then P. This order of presentation was repeated in each block of 4 days. The order of running the 8 rats in each group was varied over days so that in 4-day blocks each rat was equated for when in the running order it received its trials of the day: early (first or second), early middle (third or fourth), late middle (fifth or sixth), or late (seventh or eighth).

The 8 rats were taken in the experimental room and placed in individual holding cages. They were then divided into two units of 4 each. Each unit consisted of 2 rats from each group. The order of running the rats was as follows. A rat that was to receive the long ITI between Trials 3 and 4 received its first three trials at a 20 -sec ITI. Next, the 2 nd rat in the unit that was to receive the long ITI was run as had been the first rat. Rat 3 of the unit received its four trials at the 20-sec ITI. Then Rat 1 was given Trial 4. Next, Rat 2 of the unit was given Trial 4. This procedure produced an ITI of approximately 5 min between Trials 3 and 4 for each of the first 2 rats of the unit. Rat 4 of the unit was then given its four trials at a $20-\mathrm{sec}$ ITI. By running the second unit of 4 rats exactly as the first unit and before the first unit received its second schedule of the day, an ISI of approximately $18 \mathrm{~min}$ was produced. After the first unit received its second schedule of the day, the second unit received its second schedule. After the last of the 8 rats had been run, the rats were returned to the home cage, where they were fed the daily ration about 15 min later.

Under this procedure, olfactory cues from various sources cannot explain what the sequential-memory view predicts-that Group LP but not Group LC will run more slowly on Trials 2 and 3 of the partial reward schedule than on any other trial of either schedule. First, and most obviously, if olfactory cues associated with food reward and nonreward in the goalbox controlled responding, all rats in both groups would be expected to run rapidly on all rewarded trials and slowly on all nonrewarded trials. Second, if nonrewarded odors associated with the immediately prior trial were a signal for slow running on the current trial, all rats in both groups would be expected to run slowly on Trials 2 and 3 of the partial schedule because all rats received three consecutive nonrewarded trials in the partial schedule. Finally, earlier run rats did not supply currently run rats with differentially rewarded olfactory cues. For example, in the partial reward schedule there were two types of occasions when rats in Group LP received a trial preceded by another rat's trials These were Trial 1 (nonrewarded) and Trial 4 (rewarded). On all Trial 4 occasions and on most Trial 1 occasions, the prior trial was a rewarded one. On rare occasions, Trial 1 was not preceded by any other trial (i.e., when a rat in Group LP received the partial reward schedule and it was the 1 st rat of the day to be run). Thus it seems clear that another rat's prior rewarded trials did not differentially signal reward in Group LP, with such rewarded trials being followed about equally often by nonrewarded (Trial 1 of the partial schedule) and by rewarded (Trial 4 of the partial schedule) trials. Thus any discriminative responding shown by Group LP cannot be explained in terms of olfactory cues associated with prior reward on previous trials.

\section{Results}

In each of the three investigations reported here, a difference is said to be significant if $p<.05$. Figure 1 shows running speed of each of the two groups on each of the four trials of the consistent reward schedule and the partial reward schedule in blocks of 2 days.

In the early blocks of trials, both groups ran more slowly on the trials of the partial reward schedule than on the trials of the consistent reward schedule. With further training, two trends may be identified. First, both groups began to run as rapidly in the partial schedule as in the consistent schedule, a development especially evident on Block 4. However, with further training, Group LP but not Group LC developed the tendency to run more slowly on Trials 2 and 3 of the partial schedule than on any other trials in either schedule.

An analysis of variance (ANOVA) on the first block of trials revealed that no difference involving groups even approached statistical significance. However, running was slower in the partial schedule, or following nonrewarded trials, than in the consistent schedule, or following rewarded trials $[F(1,6)=78.80]$. The tendency of rats to run more slowly following nonrewarded than following rewarded trials early in training is a common finding (see, e.g., Capaldi, 1994).

An ANOVA over the nine blocks of trials shown in Figure 1 revealed that the group main effect was not significant $(F<1)$. However, significant differences were associated with the schedule $\times$ group interaction $[F(1,6)$ $=8.87]$, with the schedule $\times$ trial $\times$ group interaction $[F(3,18)=11.73]$, and with the schedule $\times$ trial $\times$ group $\times$ block interaction $[F(24,144)=1.83]$. Subsequent Newman-Keuls tests based on the four-way interaction revealed that on Block 9, Group LP ran significantly more slowly on Trials 2 and 3 of its partial schedule than (1) on the comparable trials of its consistent schedule and (2) Group LC on Trials 2 and 3 of either of its schedules. Group LC did not run more slowly in its partial schedule than in its consistent schedule. No other posttests were attempted.

\section{Discussion}

In the early training trials, Group LP and LC did not differ in running speed, and thus the $T_{\mathrm{O}} \mid T_{\mathrm{T}}$ ratio was at the 

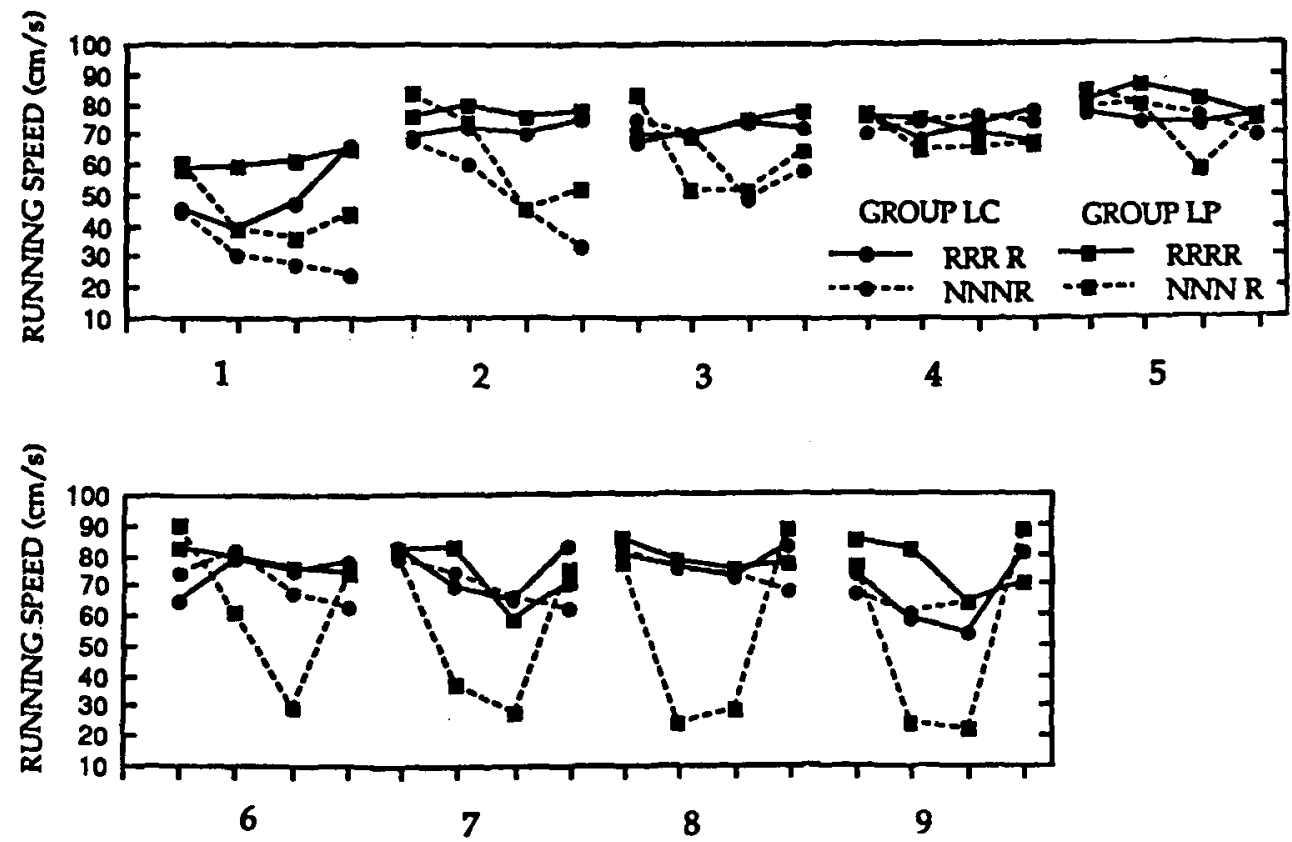

TRIALS IN EACH BLOCK OF TWO DAYS

Figure 1. Running speed of each of the two groups on each of the four trials of the consistent reward schedule and the partial reward schedule in blocks of 2 days in Experiment 1. R, rewarded; N, nonrewarded.

outset the same for both groups. This being so, differences between the groups should not have developed later in training according to SET. This prediction from SET was not confirmed. Later in training in Experiment 1, Groups LP and LC ran about equally rapidly on all trials except Trials 2 and 3 of the partial reward schedule. On those trials, Group LP ran more slowly than did Group LC. While these findings are inconsistent with SET, they are consistent with the prediction of memory-sequential views, as is shown below.

The sequential-memory view suggests that time between trials provides cues to which rats are sensitive (see, e.g., Capaldi, 1994). These time-related cues, like other cues, can become signals for reward and nonreward and they may also serve as retrieval cues. Below we provide a detailed memory analysis of the performance of Groups LP and LC on a trial-by-trial basis in each of the schedules.

In Experiment 1, cues associated with the 5-min ITI between trials (i.e., Trial 4 ) received $100 \%$ reinforcement, and so rats ran rapidly on those trials when they occurred in either the partial reward schedule or the consistent reward schedule. Cues associated either with Trial 1 of the day or with $18-\mathrm{min}$ ISI between trials received $50 \%$ irregular reinforcement in both groups and so both groups ran rapidly on those trials. However, at the 15-sec ITI, rats learned that if the prior trial was rewarded the current trial would be rewarded, and so rats ran rapidly on those trials. These trials occurred in the consistent reward schedule. Put differently, in the consistent reward schedule the memory of prior reward was a signal for reward.
It was in the partial reward schedule that the two groups learned different things. Descriptively, Group LC learned that nonrewarded trials at a relatively short ITI were sometimes rewarded, while Group LP learned that such trials were never rewarded. A more molecular analysis of such learning may be given in terms of memory retrieval. Note that initially, however, the memories of nonreward, stored on Trials 1 and 2, were retrieved respectively on Trials 2 and 3 in both groups. This is because on those trials, the storage and retrieval ITIs were the same in both groups. This being so, it follows that running speed on Trials 2 and 3 of the partial schedule would depend on how strongly the memory of nonreward became a signal for reward. This in turn would depend critically on how well the memory of nonreward was retrieved on Trial 4 of the partial reward schedule, a rewarded trial. The memory of nonreward was better retrieved on Trial 4 in Group LC than in Group LP. This is because in Group LC the ITI preceding Trial 3 (the storage trial for the memory of nonreward) and the ITI preceding Trial 4 (the critical retrieval trial for the memory of nonreward) were the same rather than, as in Group LP, different. Thus on Trials 2 and 3 of the partial schedule, the memory of nonreward was a stronger signal for reward in Group LC than in Group LP, and so on those trials Group LC ran more rapidly than did Group LP.

\section{EXPERIMENT 2}

In Experiment 2, as in Experiment 1, two groups of rats were employed, each of which received each day a four- 
trial schedule of partial reward and a four-trial schedule of consistent reward. The major noteworthy difference between Experiments 1 and 2 was this: In Experiment 2, all ITIs were about 7 min except that elapsing between Trials 3 and 4 of one of the schedules. That ITI was 20-30 sec in the consistent reward schedule for one group (Group SC), and it was 20-30 sec in the partial reward schedule group (Group SP). Thus, at the outset of training, Groups SC and SP were equated for the $T_{\mathrm{O}} \mid T_{\mathrm{T}}$ ratio.

\section{Method}

Subjects. The subjects were 8 rats of the same description as those in Experiment 1.

Apparatus. The apparatus was the same as that employed in Experiment 1 .

Pretraining. Pretraining was identical to that employed in Experiment 1.

Experimental training. All aspects of experimental training were identical to those employed in Experiment 1 except for the following. The rats were run as a single unit of 8 . The order of running the 8 rats was varied each day. With one exception, each rat received one trial of its schedule before any rat received its next trial, producing an ITI of about $7 \mathrm{~min}$. The exception to this procedure was this: Trial 4 was given $20 \mathrm{sec}$ after Trial 3 either in the partial schedule (Group SP) or in the consistent schedule (Group SC). After the first schedule had been given to each rat, the experimenter waited $20 \mathrm{~min}$ before administering the second schedule so as to produce a 20 -min ISI.

The procedure employed in Experiment 2, like that employed in Experiment 1, was such that olfactory cues from various sources cannot explain what the sequential-memory view predicts: that Group SP but not Group SC should run more slowly on Trials 2 and 3 of its partial reward schedule than on any other trial of either schedule. This is because of the following: With one exception, each rat received one trial of its schedule before any rat received the next trial; rats were run in random order over days; and all rats received the partial reward schedule 1 rat after the other, the same being true for the consistent reward schedule. If olfactory cues associated with nonreward on the prior trial influenced responding in the partial reward schedule, then, except for the first nonrewarded trial of the day, all rats in both groups should have run slowly on all nonrewarded trials, nonrewarded Trial 1 as well as nonrewarded Trials 2 and 3 . This is because except for the first nonrewarded trial of the day, each nonrewarded trial in both groups was preceded by another rat having been nonrewarded a few seconds earlier. In Group SP, Trial 4 of the partial schedule occurred shortly after nonreward, so any presumptive goalbox-related odor on that trial would be that of nonreward. Yet, rats in Group SP, as we shall see, ran rapidly on Trial 4 of the partial schedule while running slowly on Trials 2 and 3, which also followed nonreward. All other trials in both schedules occurred a few seconds after the prior rat had been rewarded. Of course, in Experiment 2, as in Experiment 1, if olfactory cues associated with food reward and nonreward in the goalbox controlled responding, all rats in both groups would be expected to run rapidly on all rewarded trials and slowly on all nonrewarded trials.

\section{Results}

Figure 2 shows running speed of each of the two groups on each of the four trials of the consistent reward schedule and the partial reward schedule in blocks of 2 days. The results obtained in Experiment 2 are quite similar to those obtained in Experiment 1. For example, on Block 1 running was much slower in the partial schedule than in the consistent schedule, with this difference becoming smaller over blocks. And by the last two blocks of trials, Group SP was running more slowly than Group LP on Trials 2 and 3 of the partial schedule.
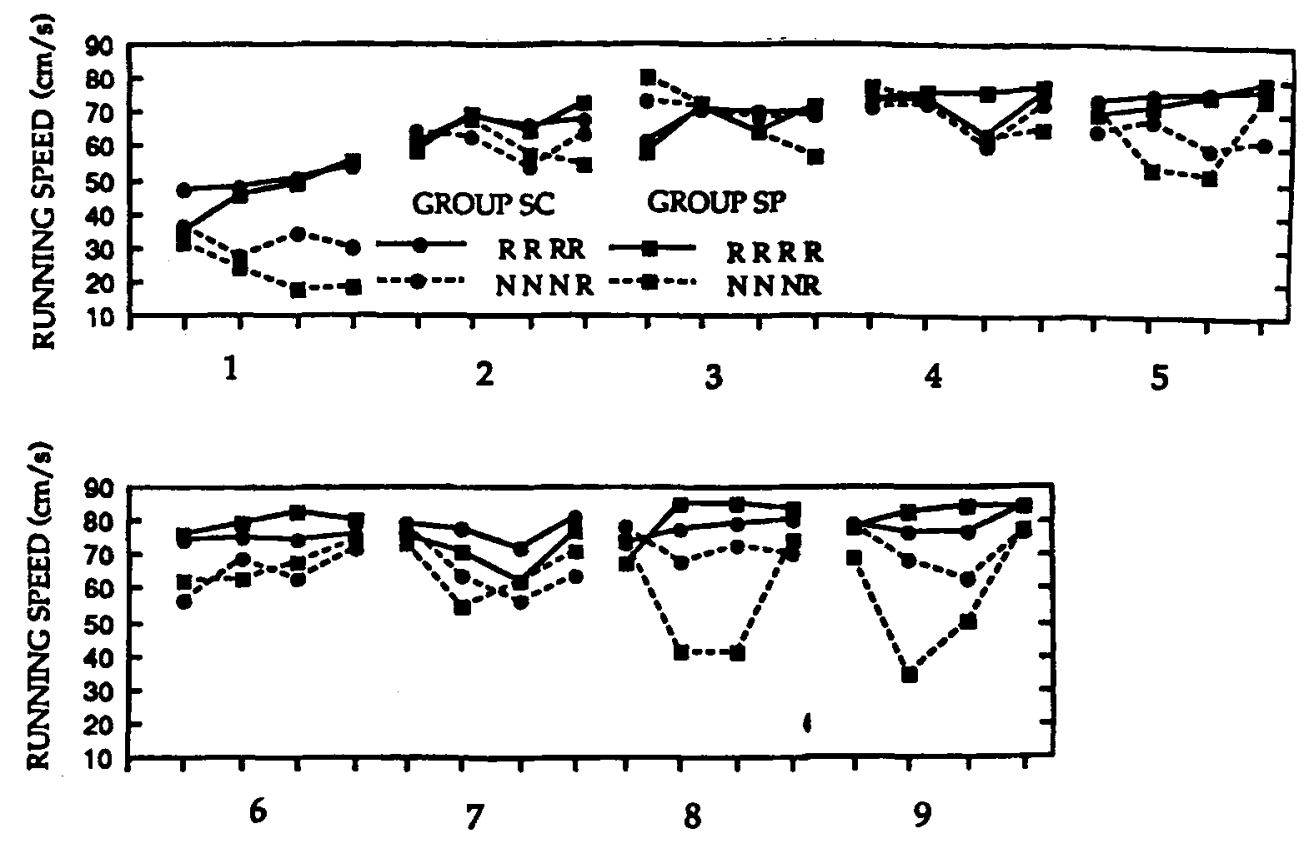

TRIALS IN EACH BLOCK OF TWO DAYS

Figure 2. Running speed of each of the two groups on each of the four trials of the consistent reward schedule and the partial reward schedule in blocks of 2 days in Experiment 2 . R, rewarded; $N$, nonrewarded. 
An ANOVA on the first block of trials revealed that no differences involving groups were significant. Running was slower in the partial schedule than in the consistent schedule $[F(1,6)=124.60]$. An ANOVA over the nine blocks of trials shown in Figure 2 revealed that the groups did not differ. However, significant differences were associated with the schedule $\times$ block $\times$ group interaction $[F(8,48)=2.20]$, with the schedule $\times$ trial $\times$ group interaction $[F(3,18)=4.84]$, and with the schedule $\times$ trial $\times$ group $\times$ block interaction $[F(24,144)=1.71]$. Subsequent Newman-Keuls tests based on the four-way interaction indicated that on Block 9, Group SP ran more slowly on Trial 2 of its partial schedule (1) than on the comparable trial of its consistent schedule and (2) than did Group SC on Trial 2 of either of its schedules. On Trial 3 of the partial schedule, Group SP ran more slowly than on Trial 3 of either consistent schedule, its own, or the consistent schedule of Group SC. However, Group SP did not run more slowly on Trial 3 of its partial schedule than did Group SC on Trial 3 of its partial schedule. Group SC did not run more slowly in its partial schedule than in its consistent schedule. No other posttests were attempted. ${ }^{1}$

\section{Discussion}

While it is difficult to make comparisons across experiments, it appears that the rats of Experiment 2 performed pretty much like those of Experiment 1 except that differences in Experiment 2 appear to be smaller. Possibly this was so because as ITI increased, running speed on nonrewarded trials increased. Consistent with this tendency, it was found here that on Trials 2 and 3 of the partial reward schedule in, for example, Block 9, Group SP of Experiment 2 (7-min ITI) ran more rapidly than did Group LP of Experiment 1 (20-sec ITI) as may be seen by comparing Figures 1 and 2. In any event, in Experiment 2 as in Experiment 1, the two groups did not differ in the early training trials and thus the $T_{\mathrm{O}} \mid T_{\mathrm{T}}$ ratio was the same in both groups. With training, however, differences between the groups developed. Group SP was definitely slower than was Group SC on Trial 2 of the partial reward schedule later in training. There was evidence that the same was true for Trial 3 as well, but the evidence was less consistent: Whereas on Trial 3 of the partial schedule, Group SP ran more slowly than on Trial 3 of either its consistent schedule or that of Group SC, it did not run more slowly than did Group SC on Trial 3 of its partial schedule. In any event, the findings of Experiment 2, like those of Experiment 1, are consistent with the sequential-memory view for exactly the same general reasons given in the discussion section of Experiment 1. Specifically, it is suggested that retrieval of the memory of nonreward on Trial 4 of the partial reinforcement schedule was poorer in Group SP than in Group SC. However, whereas in Experiment 1 this critical retrieval ITI was longer than the storage ITI, in Experiment 2 it was shorter. Descriptively, Group SC learned that nonrewarded trials at a relatively long ITI were sometimes rewarded, while
Group SP learned that such trials were never rewarded. Determining whether or not the current trial would be nonrewarded, however, rested on remembering whether the prior trial was or was not rewarded.

\section{EXPERIMENT 3}

Experiment 3 was highly similar to Experiment 1, with two noteworthy differences; all ITIs were $15 \mathrm{sec}$, and runway brightness rather than ITI was employed as the retrieval cue. In Group CP, runway brightness changed from black to white from Trial 3 to Trial 4 in the partial reinforcement schedule. In Group CC this change in brightness occurred in the consistent reinforcement schedule. Groups $\mathrm{CP}$ and $\mathrm{CC}$ had the same $T_{\mathrm{O}} \mid T_{\mathrm{T}}$ ratio at the outset of training.

\section{Method}

Subjects. The subjects were 8 rats of the same description as those in Experiments 1 and 2.

Apparatus. The apparatus consisted of two adjacent runways, identical except for brightness, one black (B), one white (W). Each runway was $194.94 \mathrm{~cm}$ long, $8.8 \mathrm{~cm}$ wide, and enclosed by $12.7-\mathrm{cm}$ sides. The runways were covered by a wire-mesh top on a hinged frame. The startbox and goalbox were $23.3 \mathrm{~cm}$ and $36.15 \mathrm{~cm}$ long, respectively, and were closed off by brass guillotine doors. Trials were timed and recorded by an IBM PC, XT Model No. 5160. A trial began with the placement of the rat in the startbox; the startbox door was raised about $3 \mathrm{sec}$ later. Raising the startbox door activated the timer, which was stopped when a photobeam, located $158.13 \mathrm{~cm}$ beyond the startbox door and $6.0 \mathrm{~cm}$ in front of the goal cup, was broken by the rat. Food ( $45 \mathrm{mg}$ Formula A Noyes pellets) could be placed in the circular goal cup $(4.0 \mathrm{~cm}$ in diameter and $1.5 \mathrm{~cm}$ in depth) cut into a wooden block $(6.5 \times 8.8 \times 4.5 \mathrm{~cm})$ located at the end of the runway. When the photobeam was interrupted, the goalbox door was lowered, confining the rat to the goalbox. If the rat had not entered the goalbox within $30 \mathrm{sec}$, the rat was placed in the goalbox, and a time of $30 \mathrm{sec}$ was assigned.

Preliminary training. On arrival at the laboratory, the rats were caged individually and given food and water ad lib for 10 days. They were then placed on food deprivation consisting of $18 \mathrm{~g}$ of Wayne lab blox each day, minus any food eaten in the apparatus. On Days 1-8 of deprivation, each rat was handled for about $1 \mathrm{~min}$. On Day 9, each rat was fed eight 45-mg Noyes pellets in the home cage. On Days 9-12, each rat was given 3 min of exploration in the B, W, $\mathrm{B}, \mathrm{W}$ alleys, respectively, and allowed to eat eight pellets scattered throughout the alley. Also, at the 1.5-min mark, the doors were lowered and raised to acquaint the rat with this noise. Following exploration the rats were fed their daily ration in their home cage. On Days 12-16, each rat received one trial in each runway (B to W on Days 13 and 15 , and $W$ to $B$ on Days 14 and 16) that terminated in food reward (R) consisting of eight 45-mg Noyes pellets. Immediately after eating the pellets, the rat was removed from the goalbox. All rats received an $R$ trial before any rat received its second $R$ trial.

Experimental training. Experimental training began on Day 17 and continued for 24 days. Trials terminated either in food reward (R) or no reward (N). On N trials, the goal cup in an adjacent gray runway was baited to control for the noise made during baiting on $\mathrm{R}$ trials, and the rats were removed from the goalbox following 15 -sec confinement. Rats were randomly assigned to two groups, and two squads of 4 rats each. Each squad consisted of 2 rats from each group. Squad 1 was always run first and the running order of rats within each squad was varied randomly on a daily basis. All rats received two different series of four trials each, RRRR and NNNR. 
For example, NNNR means that each of four responses in the runway terminated in $\mathrm{N}, \mathrm{N}, \mathrm{N}$, and $\mathrm{R}$, respectively. Group $\mathrm{CP}$ received the three successive $\mathrm{N}$ trials in the same alley, $\mathrm{B}$, and the terminal $\mathrm{R}$ trial in a different alley, $\mathrm{W}$. All trials of the RRRR series occurred in $B$. Group CC, on the other hand, received the first three $R$ trials of the RRRR series in B, and the terminal R trial of the RRRR series in W. For this group, all trials of the NNNR series occurred in $B$. Trials of a series were given in succession and were separated by about $20 \mathrm{sec}$ spent in a holding cage. There was one presentation of each series each day, the interseries interval being about $12 \mathrm{~min}$. Call the RRRR series A and the NNNR series B. The order of presenting the series each day, repeated in 4-day blocks, was AB, BA, $\mathrm{BA}$, and $\mathrm{AB}$. On Days 1 and 2 of experimental training, the rats received $A$ and $B$, respectively, which were then combined for statistical purposes and constituted Day 1 . After the last rat had been run, the 4 rats of a squad were returned to the home cage and fed the daily ration about $20 \mathrm{~min}$ later.

Under this procedure, for reasons already given in connection with Experiments 1 and 2, olfactory cues cannot explain findings of the sort expected by the sequential-memory view.

\section{Results}

Figure 3 shows running speed of each of the two groups on each of the four trials of the consistent reward schedule and the partial reward schedule in blocks of 2 days. The top portion of Figure 3 shows the first six blocks of trials, and the bottom portion shows the last six blocks. The results obtained in Experiment 3 are similar to those obtained in Experiments 1 and 2 except that performance was more variable in Experiment 3, so more training was employed in Experiment 3 than in Experiments 1 and 2. On Block 1, both groups ran more slowly in the partial schedule than in the consistent schedule. By the last block of trials, Group CP was running more slowly on Trials 2 and 3 of the partial schedule than was Group CC. However, in the middle blocks of trials, large differences apparent in one block (e.g., Block 8) might be smaller in a subsequent block (e.g., Block 10). However, the general trend from, say, Block 5 to Block 14, was for Group CP to run more slowly than Group CC on Trials 2 and 3 of the partial schedule.

An ANOVA on Block 1 revealed that no differences associated with group were significant. Running was slower in the partial schedule than in the consistent schedule $[F(1,6)=11.99]$. An ANOVA over the 12 blocks of trials shown in Figure 3 revealed that the groups did not differ $(F<1)$. However, significant differences were associated with the schedule $\times$ group interaction $[F(1,6)=$ $12.20]$, the schedule $\times$ trial $\times$ group interaction $[F(3,18)$ $=7.78]$, and with the schedule $\times$ trial $\times$ block $\times$ group interaction $[F(37,198)=1.78]$. Newman-Keuls tests based on the four-way interaction revealed the following. On Block 12, Group PC ran more slowly on Trials 2 and 3 of its partial schedule (1) than on the comparable trials of its consistent schedule and (2) than did Group CC on Trials 2 and 3 of either of its schedules. Group CC did not run more slowly in its partial schedule than in its consistent schedule. No other posttests were attempted.

\section{Discussion}

In Experiment 3, which employed brightness as the retrieval cue, results were much the same as those in Experiments 1 and 2 , which employed time between trials
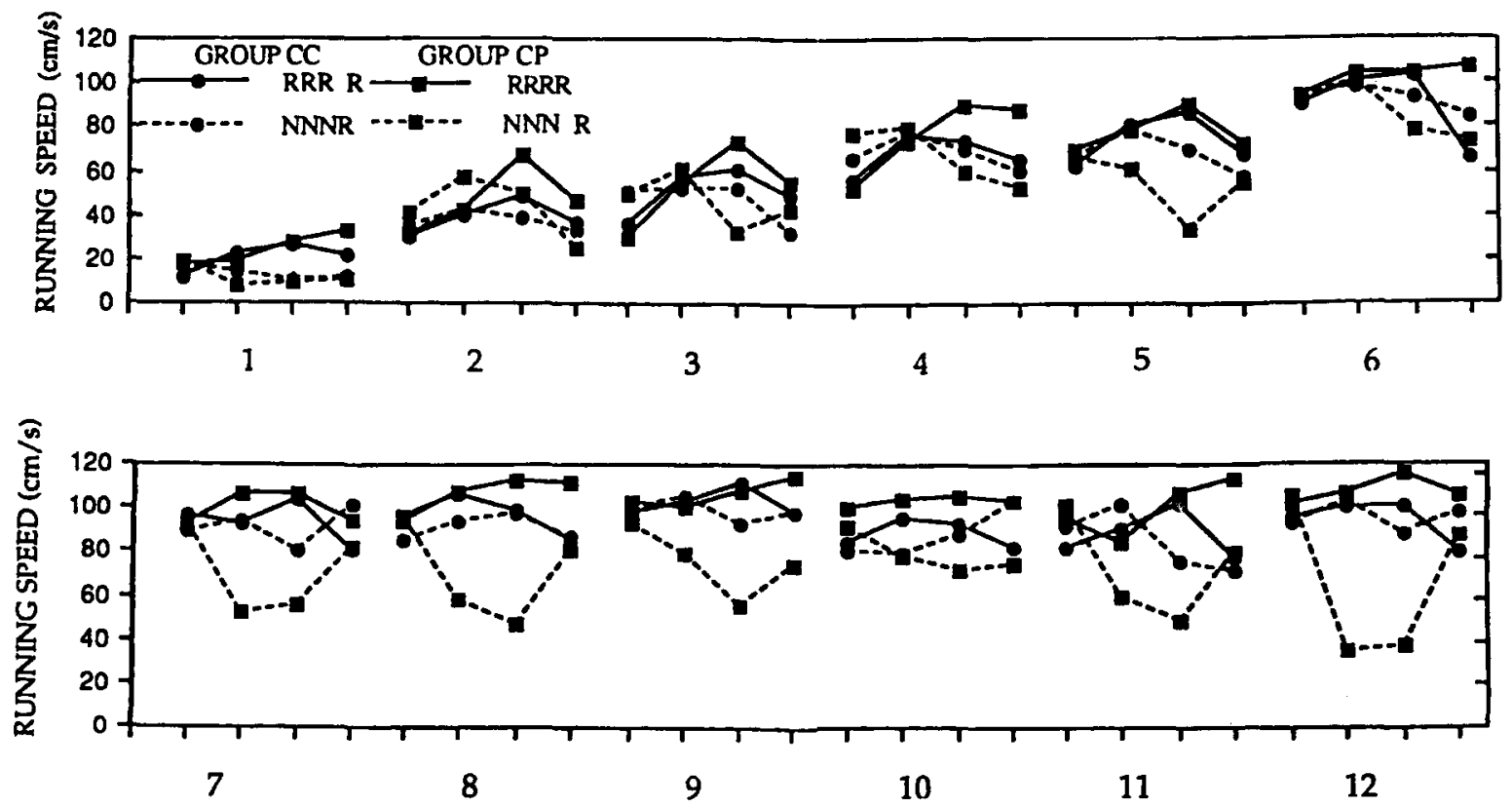

TRIALS IN EACH BI.OCK OF TWO DAYS

Figure 3. Running speed of each of the two groups on each of the four trials of the consistent reward schedule and the partial reward schedule in blocks of 2 days in Experiment 3. 
as the retrieval cue. In the early training trials of Experiment 3, the groups did not differ, and thus the $T_{\mathrm{O}} \mid T_{\mathrm{T}}$ ratio was the same in both groups. However, with training, Group CP came to run more slowly than did Group CC on Trials 2 and 3 of the partial reward schedule, with differences between the groups on other trials not being reliable. The findings obtained in Experiment 3 are inconsistent with SET and consistent with the sequentialmemory view for the same general reasons given in the discussion section of Experiment 1.

In Experiment 3 performance was variable (e.g., differences that appeared in some early block of trials might not be as large on the next block of trials). While any attempt to explain why this was so must necessarily be speculative, we suggest the following. Rats were better able to discriminate between the long and short ITIs employed in Experiments 1 and 2 than between the brightness cues employed in Experiment 3. It follows from a memory retrieval view that to the extent that the two cues are similar, memories stored in connection with one will tend to be retrieved in the presence of the other. If this hypothesis is valid, then in Group CP some elements of the memory of nonreward stored on Trial 3 of the partial reward schedule would tend to be retrieved on Trial 4. This would enable those elements to elicit the instrumental response, reducing the tendency of Group CP to run slowly on Trials 2 and 3 of the partial reward schedule. In any event, descriptively, Group CC learned that nonrewarded trials in a black runway were sometimes rewarded while Group CP learned that such nonrewarded trials were never rewarded. However, determining whether the current trial would be rewarded or nonrewarded rested on remembering whether the prior trial was rewarded or nonrewarded.

\section{GENERAL DISCUSSION}

In each of the three investigations reported here, each of two groups at the outset of training had the same $T_{\mathrm{O}} \mid T_{\mathrm{T}}$ ratio, but the memory of nonreward was a better predictor of reward in one of the groups than in the other. SET predicts that in each experiment the two groups should not differ. Contrary to SET, the group for which the memory of nonreward was the better predictor of reward ran faster than the other group on critical nonrewarded trials. The general strategy employed here to evaluate SET is similar to that employed by Durlach (1983) in the autoshaping situation using pigeons (see also, Durlach, 1989). In Durlach's investigations, which obtained results inconsistent with SET, groups that had the same waiting time to reinforcement in the presence of the target conditioned stimulus (CS) received extra reinforcers in the ITI. When these extra reinforcers were signaled by a second $\mathrm{CS}$, more responding occurred to the target CS than when the extra reinforcers were unsignaled. Thus in the present instrumental situation, as in the autoshaping situation employed by Durlach (1983), SET was disconfirmed when some stimulus (the memory of nonreward in the present case, and a second CS in Durlach's inves- tigation) was a better predictor of the reward outcome than the total time elapsing between reward outcomes.

How SET might be modified to explain the present results is not immediately clear. As a case in point, assume that in Experiments 1 and 2 timing of the following sort occurred. The rats of Groups LP and SP learned that at a given ITI, trials were rewarded if the prior trial was rewarded, but trials were nonrewarded if the prior trial was nonrewarded. This approach might have some appeal to SET since it emphasizes timing. But the view also suggests that in instrumental tasks performance is regulated by memories of goal events, a proposition explicitly rejected by SET (see Gibbon et al., 1980).

Assume for the sake of discussion that some sort of revised temporal hypothesis such as that suggested above were acceptable to SET. While such a view could explain the results of Experiments 1 and 2, it would have difficulty explaining the results of Experiment 3. First, an emphasis on timing will not explain the differences obtained in Experiment 3, since both groups were both rewarded and nonrewarded at short ITIs following either reward or nonreward. To explain the result of Experiment 3 , remembered brightness must be brought in to the explanatory mix, and, moreover, brightness cues must be seen as having properties similar to those of temporal cues. These developments complicate considerably the propositions that time is a unique cue and that to understand instrumental runway performance it is sufficient to know the $T_{\mathrm{O}} \mid T_{\mathrm{T}}$ ratio.

The runway results reported here clearly differ from those reported by Gibbon et al. (1980) employing pigeons in an autoshaping situation. The difference between the two sets of results could be due to the numerous differences that necessarily exist between the present instrumental runway situation and the autoshaping situation. On this view the runway and autoshaping situations are simply not comparable. While that possibility cannot be and possibly should not be discounted, there is another alternative that also deserves to be taken seriously: At each ITI in the Gibbon et al. (1980) investigation, groups that differed in percentage of reward performed similarly, not because of SET, but for a variety of different reasons. This hypothesis is viable because those groups, while equated along the ratio dimension deemed important by SET, simultaneously differed along a number of other dimensions. The groups compared here were equated not only along the dimensions that varied in the Gibbon et al. (1980) investigation, but also along a variety of additional dimensions, as will become clear below.

In many varied reward investigations of the sort reported here, groups that differ along some explicitly manipulated dimension almost invariably differ along a number of other unwanted dimensions. For example, if percentage of reinforcement is varied and, as often happens in the instrumental area, number of acquisition trials is equated, the groups will differ in terms of number of rewarded trials, number of nonrewarded trials, and sequence of rewarded and nonrewarded trials (see, e.g., Capaldi, 1994; Capaldi et al., 1986). However, if groups 
receiving different percentages of reward are equated along some other dimension, other unwanted and possibly important differences between the groups may be introduced. For example, Gibbon et al. (1980) varied reward percentage while equating groups for number of reinforcements. This procedure results in lower percentage of reward groups receiving a greater number of nonrewarded trials, a greater number of total trials, and, of considerable importance, we would suggest (see, e.g., Capaldi, 1994), different sequences of rewarded and nonrewarded trials.

A prime objective of the three investigations reported here was to equate groups for the $T_{\mathrm{O}} \mid T_{\mathrm{T}}$ ratio. This objective, desirable in itself, had another desirable effect. It develops that when groups are equated for the $T_{\mathrm{O}} \mid T_{\mathrm{T}}$ ratio as here, employing the two schedules procedure, they are also equated along a number of other dimensions, an unprecedented number for investigations of the present type. For example, the groups employed here in each investigation were equated for overall percentage of reward, for sequence of rewarded and nonrewarded trials, for number of rewarded trials and number of nonrewarded trials, and for number of long and/or short ITIs both within a day and between days.

Also, the groups were equated for some other variables, which while not as yet systematically applied to varied reward situations, have been suggested as exercising influence in certain cases of varied reward (see D'Amato, 1991). For example, the groups compared here received not only the same percentage of reward overall, but also the same percentage of reward at each ordinal position of trial (e.g., Trial 1, Trial 2, etc.), the ordinal position variable being seen as controlling instrumental responding in some situations, including some varied reward situations (see, e.g., D'Amato, 1991). The most obvious example of such control occurred in connection with Experiment 3. In Experiment 3, both groups received 50\% reward in connection with trial positions 1,2 , and 3 of the day and $100 \%$ reward in connection with trial position 4 .

While the present investigations were explicitly designed with SET in mind, it develops that the control procedures employed to accomplish a test of that view and the sequential view produced findings that are relevant to a variety of other theories as well. This is so because one or another of the dimensions along which the groups were equated here is considered of importance to one or another theory. Thus, those theories, like SET, encounter difficulty in explaining why in each investigation the groups examined here differed as they did. This has already been shown to be the case with respect to the ordinal position of trial view favored by D'Amato (1991). As another example, groups receiving the same number of rewarded and nonrewarded trials, as here, should be equally frustrated according to frustration theory and so should perform similarly (see Amsel, 1958, 1992). That is, frustration theory cannot explain why some of the groups employed here responded differentially on rewarded and nonrewarded trials. Note first that no external source of stimulus control, such as brightness cues, was differentially rewarded in a manner that would support the discriminative responding observed here. To explain such discriminative responding, some source of internal stimulus control must be postulated (e.g., retrieved memories). But, a source of internal stimulus control capable of explaining the present findings does not exist within frustration theory. It is the case that an internal stimulus, a rapidly fading stimulus trace or aftereffect of nonreward that was said to persist for about $60 \mathrm{sec}$ in the weanling rat, was recently identified by Amsel (1994). Rapidly fading aftereffects, unlike retrieved memories, cannot explain the findings obtained here. In Experiment 2, for example, rapidly fading aftereffects could not have served as the source of differentially rewarded stimuli. This is because neither in Group SP nor in Group SC would aftereffects have been present on Trials 2 and 3 of the partial reward schedule because those trials were separated by a 7-min ITI. Moreover, in Experiment 3 all trials occurred at a short 20 -sec ITI and both groups received exactly the same schedule of rewarded and nonrewarded trials. Thus in Experiment 3, aftereffects of nonreward would have acquired an identical capacity to elicit responding in both groups and thus could not explain differences between them. We suggest that it will not be easy either for some other theory to explain the results reported without reference to some sort of memory assumption. In short, the present results supply exceptionally strong support for the view that goal-produced memories regulate instrumental responding.

\section{REFERENCES}

AMSEL, A. (1958). The role of frustrative nonreward in noncontinuous reward situations. Psychological Bulletin, 55, 102-119.

Amsel, A. (1992). Frustration theory: An analysis of dispositional learning and memory. New York: Cambridge University Press.

Amsel, A. (1994). Endnotes. Psychonomic Bulletin \& Review, 1, 327-332.

CAPALDI, E. J. (1994). The sequential view: From rapidly fading stimulus traces to the organization of memory and the abstract concept of number. Psychonomic Bulletin \& Review, 1, 156-181.

CaPaldi, E. J., Nawrocki, T. M., Miller, D. J., \& Verry, D. R. (1986). Time between events as a retrieval cue: Recall and the temporal similarity between the storage and retrieval intervals. Journal of Experimental Psychology: Animal Behavioral Processes, 12, 258-269.

D'Amato, M. R. (1991). Comparative cognition: Processing of serial order and serial pattern. In L. Dachowski \& C. F. Flaherty (Eds.), Current topics in animal learning: Brain, emotion, and cognition (pp. 165-185). Hillsdale, NJ: Erlbaum.

DuRLACH, P. J. (1983). Effect of signaling intertrial unconditioned stimuli in autoshaping. Journal of Experimental Psychology: Animal Behavior Processes, 9, 374-389.

DuRLaCH, P. J. (1989). Learning and performance in Pavlovian conditioning: Are failures of contiguity failures of learning or performance? In S. B. Klein \& R. R. Mowrer (Eds.), Contemporary learning theories: Pavlovian conditioning and the status of traditional learning theory (pp. 19-59). Hillsdale, NJ: Erlbaum.

Gallistel, C. R. (1994). Space and time. In N. J. Mackintosh (Ed.), Animal learning and cognition (pp. 221-253). San Diego: Academic Press.

Gibbon, J., \& Balsam, P. (1981). Spreading associations in time. In C. M. Locurto, H. S. Terrace, \& J. Gibbon (Eds.), Autoshaping and conditioning theory (pp. 219-253). New York: Academic Press.

Gibbon, J., Farrell, L., Locurto, C. M., Duncan, H. J., \& Terrace, H. S. (1980). Partial reinforcement in autoshaping with pigeons. $A n-$ imal Learning \& Behavior, 8, 45-59. 


\section{NOTE}

1. The memory of nonreward is progressively modified over successive nonrewarded trials (see, e.g., Capaldi, 1994). Thus, even in the groups in which the storage and retrieval ITI were the same in the partial schedule (Groups LC and SC), it would still be possible to learn the discrimination problem. That is, in those groups the rewarded memory of nonreward ( $\mathrm{S}+\mathrm{cue}$ ) signaling reward on Trial 4 of the partial sched- ule is somewhat different from the nonrewarded memories of nonreward ( $\mathrm{S}$ - cues) signaling nonreward on Trials 2 and 3 of the partial schedule. This factor may account for the somewhat slow running of Group SC on Trial 3 of its partial reward schedule in Block 9.

(Manuscript received January 3, 1995; revision accepted for publication April 2, 1995.) 